\title{
Investigación de las Concepciones Alternativas sobre Ácidos y Bases en Estudiantes de Secundaria
}

\author{
Investigating the Alternative Conceptions on Acids and Bases in Secondary students
}

\author{
Flor M. Jiménez-Aponte, Manuel F. Molina, José G. Carriazo* \\ Departamento de Química, Facultad de Ciencias, Universidad Nacional de Colombia, Carrera 30 No. 45-03, Bogotá- \\ Colombia. \\ *To whom the correspondence should be addressed: jcarriazog@unal.edu.co
}

\begin{abstract}
Resumen- Este trabajo presenta la exploración de las concepciones alternativas, sobre los conceptos de ácido y base, en estudiantes de grados octavo y noveno de educación secundaria. La información establece de manera concreta la necesidad de intervenir la ejecución del currículo con estrategias que mejoren la integración del conocimiento cotidiano con el saber científico alrededor de los conceptos de ácido y base. Se observó el aislamiento de los conceptos estudiados con el lenguaje químico, la reactividad química y los procesos biológicos fundamentales.
\end{abstract}

Palabras clave: Concepciones alternativas, ácido-base, acidez, basicidad, aprendizaje en química.

\begin{abstract}
This work shows the exploration of alternative conceptions, on both acid and base concepts, of 8th and 9th grade secondary students. The obtained results clearly establish the need of intervening the curriculum execution, using educational strategies that can improve the integrating of everyday knowledge with scientific knowledge about acid and base concepts. An isolation of these studied concepts regarding chemistry language, chemical reactivity and fundamental biological processes was observed.
\end{abstract}

Key Word: alternative conceptions, acid-base, acidity, basicity, chemistry learning.

\section{INTRODUCCIÓN}

En el currículo de los grados octavo y noveno de la educación básica secundaria en Colombia, uno de los temas pertinentes para el estudiante contemporáneo es el que involucra los conceptos de ácidos y bases; conceptos que posibilitan explicar fenómenos de la vida cotidiana [1], puesto que muchos de los productos que se consumen ostentan un carácter ácido o un carácter básico, lo que genera la necesidad de fundamentar un cambio metodológico en la enseñanza de la química, para que los alumnos estructuren estos conceptos con ideas científicas y no sólo con los conocimientos que le proporciona el uso cotidiano de los productos mencionados. El entendimiento de estos conceptos incide en la calidad de

Fecha de Recepción: 24 de Abril de 2014

Fecha de Aceptación:22 de Junio de 2015 vida y contribuye a la búsqueda de soluciones a problemas globales que son consecuencia del desarrollo científico y tecnológico [2].

A. Una introducción al componente históricoepistemológico:

Los conceptos de ácido y base han pasado por diferentes etapas, que se remontan desde la época primitiva hasta nuestros días, pasando de una caracterización netamente sensorial y por sus propiedades organolépticas hasta los conceptos actuales de Arrhenius, Bronsted-Lowry, Lewis, Pearson y las propuestas conceptuales de Usanovich y de Lux y Flood.

Las culturas Mesopotámica y Egipcia en el año 640 d. de C, comienzan su participación, no estudiando ni definiendo los ácidos y las bases, sino empleándolos de forma empírica en la metalurgia. Utilizaron mezclas de ácido nítrico y ácido clorhídrico para disolver el oro, fundamentando este comportamiento en situaciones "divinas" y no en conceptos químicos [3, 4]. Luego de algunas contribuciones de la cultura árabe y de la cultura griega, en los siglos XVI y XVII se retoman los conceptos de ácido y base pero aplicados de forma cualitativa a los procesos metabólicos; es el caso de Van Helmont (1577-1644) quien reconoció un licor ácido presente en el estómago como el encargado del desdoblamiento de los alimentos, iniciándose con esto el estudio de los procesos bioquímicos. Posteriormente, Franciscus Sylvius de le Boe (1614-1672) continuó los estudios y afirmó que, en los procesos químicos ocurridos en el interior de los organismos, actuaban sustancias particulares denominadas las "acideces y los alcalinidades reales"; convirtiéndose en el precursor del concepto moderno de $\mathrm{pH}$ [5].

Posteriormente, el químico Lémery (1645-1715) basado en la teoría corpuscular de Isaac Newton, indicó que los ácidos estaban formados por corpúsculos o partículas puntiagudas que punzaban la lengua, y que las bases tenían zonas de poros por donde las puntas de los ácidos podían penetrar, formando 
una sustancia diferente a la inicial: una sal neutra [6]. Por otra parte, Antoine Laurent Lavoisier (1743- 1749) descubrió que al disolver ciertos óxidos de no metales en agua se obtiene una solución ácida, lo que lo llevó a concluir que el oxígeno es un elemento común a todos los ácidos y que las propiedades de los mismos son consecuencia de la presencia de este elemento [3]. Humphry Davy, en 1811, luego de muchos experimentos, encuentra que el ácido muriático (ácido clorhídrico) no contenía oxígeno y en 1814 publica qué: "la acidez no depende de una sustancia elemental particular, sino de una ordenación peculiar de varias sustancias". Gracias a esto, se le adjudica la responsabilidad de establecer que, un ácido está asociado a la presencia del ion hidrógeno $\left(\mathrm{H}^{+}\right)$y no al oxígeno, convirtiendo esta observación en la nueva idea de los conceptos de ácido y base. Además, Michael Faraday estudió la disociación de los ácidos, las bases y las sales, en partículas cargadas y concluyó que estas sustancias son electrólitos, que a su vez se pueden clasificar como fuertes, cuando la corriente eléctrica pasa fácilmente a través de ellos o débiles cuando se les dificulta hacerlo.

En el año 1884, Arrhenius clasificó los ácidos y las bases como sustancias que están en capacidad de liberar iones hidrógeno $\left(\mathrm{H}^{+}\right)$o liberar iones hidroxilo $\left(\mathrm{OH}^{-}\right)$en el agua, respectivamente [7] y estableció que al presentarse la disociación en fase acuosa, la mezcla se convertiría en conductora de corriente eléctrica. En el año de 1923, J. N. Bronsted y Thomas Lowry definen los ácidos como sustancias donadoras de protones $\left(\mathrm{H}^{+}\right)$y las bases como aceptoras de los mismos, extendiendo el concepto más allá de las soluciones acuosas y ampliándolo a sistemas no acuosos sin el requerimiento de grupos hidroxilo para las bases. También en el año de 1923, el químico norteamericano Gilbert N. Lewis propuso conceptos más amplios y útiles sobre ácidos y bases. Lewis definió las bases como sustancias que pueden donar un par de electrones, y los ácidos como sustancias que pueden aceptar ese par electrónico [7]. Esta definición tampoco ofrece un criterio absoluto de clasificación de una sustancia como ácida, neutra o básica sino que siempre dependerá de la sustancia con las que se compare [6].

Luego, en el año 1939, Lux y Flood definen los ácidos como sustancias capaces de aceptar iones óxido, y las bases como sustancias que pueden ceder estos iones [6, 8]; definición que para los ácidos y las bases está basada en procesos restringidos de transferencia de iones. Esta definición indica la posibilidad de reacción directa entre óxidos ácidos y óxidos básicos, bajo las condiciones apropiadas. En ese mismo año, 1939, M. Usanovich, teniendo en cuenta los procesos de transferencia de iones, cationes o aniones e incluso electrones, propone de manera más general que las bases son sustancias capaces de donar aniones o electrones, o que pueden aceptar cationes; y que los ácidos son sustancias capaces de ceder cationes o combinarse con aniones o electrones [6]. Evidentemente las definiciones de Usanovich incluyen las reacciones de óxido-reducción en el campo de la química ácido-base [8] y contienen de manera más generalizada las ideas planteadas por Lux y Flood. En la actualidad las definiciones de Lux y Flood o las de Usanovich son poco empleadas en química, quizá porque se reducen a reacciones de transferencia de iones.

Por otra parte, a principios de la década de los setenta, R. Pearson basado en las definiciones de Lewis introdujo los conceptos de ácidos y bases duros y blandos, ante la necesidad de explicar las tendencias preferenciales de unión entre iones metálicos y ligantes que formarían complejos de coordinación [7, 8]. Según Pearson, los ácidos duros son especies químicas aceptoras de pares de electrones, compactas, con tamaño pequeño y carga elevada. Las bases duras son especies químicas donadoras de pares de electrones, de tamaño pequeño y muy electronegativas [7, 9]. Los ácidos blandos y bases blandas son especies químicas de mayor tamaño y fácilmente polarizables. En este contexto, los iones $\mathrm{Al}^{3+}, \mathrm{Cr}^{3+}, \mathrm{H}^{+}, \mathrm{Fe}^{3+}$, son ejemplos de ácidos duros; los iones $\mathrm{F}^{-}$ , $\mathrm{Cl}^{-}, \mathrm{OH}^{-}, \mathrm{NH}_{3}$, son ejemplos de bases duras; mientras que $\mathrm{Cu}^{2+}, \mathrm{Ag}^{+}, \mathrm{Cd}^{2+}, \mathrm{Au}^{+}$, son ácidos blandos; $\mathrm{CN}^{-}, \mathrm{CO}, \mathrm{I}^{-}, \mathrm{S}_{2} \mathrm{O}_{3}=$, son bases blandas [7, 9].

Las descripciones anteriores muestran la evolución y la complejidad en la construcción de los conceptos de ácido y base. En la actualidad, las definiciones más importantes y útiles en química son las proporcionadas por Bronsted-Lowry, Lewis y Pearson. Sin embargo, no se pretende que los estudiantes logren el dominio de la complejidad conceptual de dicha temática, pero sí es necesario que los profesores posean dichos conceptos con claridad y entendimiento.

\section{B. Una introducción al componente didáctico:}

Investigaciones acerca del aprendizaje de las ciencias han demostrado que los alumnos sin importar el nivel académico en el que se encuentran, han tenido procesos cognitivos previos con los cuales han formado conceptos propios sobre la ciencia y el conocimiento científico, este proceso cognitivo puede deberse a experiencias cotidianas, creencias populares, conocimientos de instituciones anteriores o a un simple descubrimiento casual o experimental que los lleva a formar sus propias ideas de dicho tema: debido a este conocimiento y basados en su aprendizaje significativo, los ha llevado a crear sus propias concepciones, adecuadas o inadecuadas, acerca del conocimiento científico [10]. Todo lo anterior influye en la creación de las concepciones alternativas [11], que son construcciones que los sujetos elaboran para dar respuesta a su necesidad de interpretar fenómenos naturales, y aunque estas puedan ser ideas equivocadas, se convierten en un rápido esquema mental [12] que para cada persona explica y resuelve un problema que luego entra en conflicto con lo que la ciencia acepta. Es aquí donde se requiere la intervención del docente para ayudar a modificar estas ideas previas de los estudiantes. 
La identificación y modificación de las ideas previas que podrían tener los estudiantes es un paso fundamental para el proceso de enseñanza-aprendizaje, donde no sólo aprende el alumno sino también el docente, porque es éste quien debe poseer una comprensión global y contextualizada sobre la realidad del concepto, que le permita después introducirlo en un lenguaje educativo entendible para el estudiante, con lo que rutinariamente también se entera de situaciones o ideas que quizás no había identificado antes sobre determinado tema [13].

Las concepciones alternativas son difíciles de modificar y pueden permanecer de generación en generación, lo que ha hecho que en las últimas décadas, diferentes autores a nivel mundial, se hayan dedicado a investigar sobre estos fenómenos del pensamiento o del aprendizaje en diferentes áreas del conocimiento. En algunos casos se han evaluado las concepciones alternativas sobre los conceptos de ácido y base que presentan los estudiantes y docentes en los diferentes niveles educativos (primaria, secundaria y universidad) [6].

En España, en el año 2002, se adelantó un trabajo con estudiantes de Licenciatura en Química, sobre los conceptos de ácido y base que manejaban y las dificultades más frecuentes que ellos tenían [14], con el objetivo de dar herramientas a los docentes para establecer metodologías tendientes a responder a las dificultades identificadas y facilitar el aprendizaje de los estudiantes españoles. En España y México, Carlos Furio y colaboradores, en el año 2007, presentaron un estudio realizado a los estudiantes de último grado de bachillerato, para establecer si dominaban las conceptualizaciones macroscópica y microscópica del comportamiento ácido-base y la relación entre diferentes modelos conceptuales. Como resultado, encontraron que los estudiantes presentaban un marco conceptual pobre sobre los ácidos y las bases, por ende no podían interpretar las propiedades de estas sustancias [2]. En el 2009, Yezdan [15], en la Universidad Estatal de Oklahoma (Estados Unidos), realizó un estudio con futuros profesores de química, para determinar las ideas previas que éstos tenían sobre los conceptos de ácido y base y observar las diferencias que se presentaban entre el concepto y la aplicación del mismo, pasando de la teoría a la práctica. Como resultado, encontró que los futuros docentes no manejaban con propiedad los conceptos fundamentales de la química general, entre ellos los conceptos de ácido y base evidenciando falencias en sus programas de formación [15]. En Tailandia, Artdej y colaboradores [16], realizaron una prueba diagnóstica a estudiantes de grado once, el ABDT (Acid-Base Diagnostic Test) y encontraron que aunque los estudiantes tenían conceptos propios y conceptos dados por sus profesores de química, tenían menos dificultad en llevar a la práctica este conocimiento que en entender el fundamento teórico del mismo [16]. La conclusión de este estudio permitió establecer un método de enseñanza eficaz que permitiera ser utilizado en la educación para la enseñanza de los conceptos tanto teóricos como prácticos en área de la química [16].

McClary y Talanquer [17] caracterizaron los modelos mentales de los ácidos y las ideas de fuerza ácida en un grupo de estudiantes universitarios de primer semestre de un programa de química. Pero la literatura sugiere que simplemente la identificación y clasificación de las concepciones alternativas no es suficiente para mejorar el aprendizaje de los estudiantes, sino que se requiere además investigar sobre las ayudas didácticas necesarias para lograr el cambio conceptual en los estudiantes y desplazar su pensamiento desde sus conceptos iniciales a los conceptos más concordantes con la visión científica [18].

Diversas investigaciones referencian las principales dificultades en la enseñanza y el aprendizaje de los conceptos de ácidos y bases, vinculando las ideas previas o concepciones alternativas de los estudiantes $[13,14,16,19$, 20]. Sin embargo, en dichas investigaciones poco se contemplan los alcances y las limitaciones contextualizadas de los conceptos de ácido y base. En este sentido, es relevante generar información concreta que permita entender y aproximar las ideas previas de los estudiantes hacia los conceptos de acidez y basicidad y sus implicaciones en el mundo biológico y social, con la finalidad de acercarse fielmente a los conceptos científicos, en el marco de la propuesta de un cambio conceptual y teniendo en cuenta las tendencias actuales de alfabetización tecno-científica, permitiendo orientar la educación escolar hacia un verdadero aprendizaje significativo.

En el presente trabajo se reconocen las concepciones alternativas, de un grupo de estudiantes de los grados octavo y noveno de la zona quinta de Usme (Bogotá, D.C., Colombia), sobre los conceptos de ácidos y bases. La información obtenida permite avanzar en el marco investigativo que pretende determinar en qué medida las concepciones alternativas interfieren en el aprendizaje de esta temática y cómo se relacionan con el entorno social.

\section{METODOLOGÍA}

El instrumento utilizado fue un cuestionario (Figura 1) de 20 preguntas de selección múltiple con única respuesta, divididas en siete temáticas (Tabla 1) que permiten relacionar la información cotidiana de los estudiantes con los intereses de la indagación. Se aplicó a 111 estudiantes del colegio Brazuelos (año 2011), localidad quinta-USME (BogotáColombia), con promedio de edad entre los 13 y 15 años, quienes cursaban los grados octavo y noveno y que además tuvieron dentro su Plan Curricular el estudio de los conceptos de ácido y base. 


\begin{tabular}{|c|c|c|c|c|}
\hline PREGUNTA & A & B & C & D \\
\hline $\begin{array}{l}\text { 1. Los ácidos al reaccionar con metales, pueden } \\
\text { desprender }\end{array}$ & Oxígeno & Carbono & Hidrógeno & Nitrógeno \\
\hline 2. Los alimentos ácidos tienen un sabor & Dulce & Agrio & Amargo & Salado \\
\hline 3. Los ácidos al disolverse en agua producen iones de & Calcio & Hidrógeno & Carbono & Oxígeno \\
\hline 4. De las siguientes sustancias, es ácida & $\mathrm{NaOH}$ & $\mathrm{H}_{2} \mathrm{O}$ & $\mathrm{HBr}$ & $\mathrm{NH}_{3}$ \\
\hline 5. Un material de naturaleza ácida utilizada en casa es: & $\begin{array}{l}\text { Leche de } \\
\text { magnesia }\end{array}$ & Aspirina & Ajax & Shampú \\
\hline $\begin{array}{l}\text { 6. Una sustancia básica al contacto con la piel da la } \\
\text { sensación de: }\end{array}$ & Abrasiva & Áspera & Rugosa & Resbalosa \\
\hline $\begin{array}{l}\text { 7. Un material de naturaleza básica utilizado a diario en } \\
\text { casa es: }\end{array}$ & Jabón & Vinagre & Alcohol & $\begin{array}{c}\text { Agua } \\
\text { oxigenada }\end{array}$ \\
\hline 8. Los alimentos básicos tienen un sabor: & Dulce & Agrio & Amargo & Salado \\
\hline 9. Las bases al disolverse en agua producen iones: & Hidrógeno & Hidroxilo & Fósforo & Oxígeno \\
\hline 10. Es una sustancia básica: & $\mathrm{NaOH}$ & $\mathrm{CH}_{3} \mathrm{COOH}$ & $\mathrm{H}_{3} \mathrm{PO}_{4}$ & $\mathrm{H}_{2} \mathrm{SO}_{4}$ \\
\hline $\begin{array}{l}\text { 11. Los problemas de acidez estomacal, se presentan por } \\
\text { exceso de: }\end{array}$ & $\mathrm{HCl}$ & $\mathrm{CO}_{2}$ & $\mathrm{H}_{2} \mathrm{O}$ & $\mathrm{CaCO}_{3}$ \\
\hline $\begin{array}{l}\text { 12. En la preparación de algunos alimentos se usa una } \\
\text { sustancia de carácter básico, ella es: }\end{array}$ & $\begin{array}{l}\text { Bicarbonato } \\
\text { de sodio }\end{array}$ & $\begin{array}{c}\text { Hidróxido } \\
\text { de } \\
\text { aluminio }\end{array}$ & $\begin{array}{c}\text { Carbonato } \\
\text { de calcio }\end{array}$ & $\begin{array}{l}\text { Hidróxido } \\
\text { de Sodio }\end{array}$ \\
\hline $\begin{array}{l}\text { 13. En los seres humanos es fundamental mantener } \\
\text { constante el pH de la sangre, para ello participa el: }\end{array}$ & $\begin{array}{c}\text { Ácido } \\
\text { clorhídrico }\end{array}$ & $\begin{array}{l}\text { Ácido } \\
\text { carbônico }\end{array}$ & $\begin{array}{l}\text { Ácido } \\
\text { láctico }\end{array}$ & $\begin{array}{l}\text { Ácido } \\
\text { acético }\end{array}$ \\
\hline $\begin{array}{l}\text { 14. Un componente de carácter básico en fluidos de } \\
\text { nuestro cuerpo es la UREA, ella se encuentra en la: }\end{array}$ & Saliva & Orina & Linfa & Sangre \\
\hline $\begin{array}{l}\text { 15. En la fabricación de panes y pasteles, se utiliza polvo de } \\
\text { hornear que contiene: }\end{array}$ & Bicarbonato & $\begin{array}{l}\text { Jugo de } \\
\text { limón }\end{array}$ & Vinagre & $\begin{array}{c}\text { Conserva } \\
\text { nte }\end{array}$ \\
\hline $\begin{array}{l}\text { 16. Durante la práctica de deporte como el atletismo, el } \\
\text { futbol, la natación, entre otros, los músculos producen: }\end{array}$ & $\begin{array}{c}\text { Ácido } \\
\text { sulfúrico }\end{array}$ & $\begin{array}{c}\text { Ácido } \\
\text { clorhídrico }\end{array}$ & $\begin{array}{l}\text { Ácido } \\
\text { láctico }\end{array}$ & $\begin{array}{l}\text { Ácido } \\
\text { cítrico }\end{array}$ \\
\hline $\begin{array}{l}\text { 17. De los siguientes elementos el que forma un óxido } \\
\text { básico al combinarse con oxígeno es: }\end{array}$ & Azufre & Silicio & Cloro & Cobre \\
\hline $\begin{array}{l}\text { 18. La limonada y el jugo de frutas como naranja y } \\
\text { mandarina contiene: }\end{array}$ & $\begin{array}{l}\text { Ácido } \\
\text { acético }\end{array}$ & $\begin{array}{c}\text { Ácido } \\
\text { propiónico }\end{array}$ & $\begin{array}{l}\text { Ácido } \\
\text { cítrico }\end{array}$ & $\begin{array}{c}\text { Ácido } \\
\text { etanoico }\end{array}$ \\
\hline 19. Cuál de estos materiales cree Usted que NO es básico: & Diablo rojo & Vinagre & Cemento & Milanta \\
\hline $\begin{array}{l}\text { 20. De los siguientes elementos el que forma un óxido } \\
\text { ácido al combinarse con oxigeno es: }\end{array}$ & Sodio & Calcio & Carbono & Aluminio \\
\hline
\end{tabular}

Figura 1. Cuestionario aplicado a los estudiantes para conocer sus concepciones alternativas. 
Tabla 1. Caracterización de los ítems del cuestionario sobre ideas previas.

\begin{tabular}{|c|c|}
\hline Temáticas-Información Buscada & $\begin{array}{l}\text { Pregunta } \\
\text { relacionada }\end{array}$ \\
\hline $\begin{array}{l}\text { Reacciones que presentan los ácidos y las } \\
\text { bases }\end{array}$ & $1,3,9,17,20$ \\
\hline $\begin{array}{l}\text { Propiedades organolépticas de los ácidos y de } \\
\text { las bases }\end{array}$ & $2,6,8$ \\
\hline $\begin{array}{l}\text { Diferenciación entre un ácido y una base por } \\
\text { su nomenclatura }\end{array}$ & 4,10 \\
\hline $\begin{array}{l}\text { Identificación de los ácidos y las bases en las } \\
\text { sustancias y alimentos de uso diario }\end{array}$ & $5,7,12,18,19$ \\
\hline $\begin{array}{l}\text { Reconocimiento de la importancia de los } \\
\text { ácidos y las bases en el cuerpo humano }\end{array}$ & $11,13,14,16$ \\
\hline $\begin{array}{l}\text { Reconocimiento de las sustancias básicas en } \\
\text { el uso industrial }\end{array}$ & 15 \\
\hline $\begin{array}{l}\text { Composición química de los ácidos y las } \\
\text { bases }\end{array}$ & 4,10 \\
\hline
\end{tabular}

\section{RESULTADOS Y DISCUSIÓN}

Los resultados obtenidos se resumen en la Tabla 2.

Tabla 2. Resultados obtenidos de la aplicación de cuestionario para explorar las concepciones alternativas (Colegio Brazuelos Grados Octavo y Noveno).

\begin{tabular}{|c|c|c|c|c|}
\hline \multirow[t]{2}{*}{ Preguntas } & \multicolumn{4}{|c|}{$\begin{array}{l}\text { Valoración de cada respuesta } \\
(\%, \text { aproximado al entero más cercano) }\end{array}$} \\
\hline & A & B & $\mathrm{C}$ & D \\
\hline 1 & 23 & 25 & 27 & 25 \\
\hline 2 & 2 & 52 & 41 & 5 \\
\hline 3 & 21 & 37 & 25 & 17 \\
\hline 4 & 43 & 7 & 23 & 26 \\
\hline 5 & 30 & 24 & 31 & 15 \\
\hline 6 & 11 & 29 & 15 & 45 \\
\hline 7 & 50 & 18 & 12 & 21 \\
\hline 8 & 39 & 9 & 13 & 39 \\
\hline 9 & 35 & 23 & 14 & 27 \\
\hline 10 & 37 & 15 & 14 & 34 \\
\hline 11 & 32 & 29 & 7 & 32 \\
\hline 12 & 68 & 3 & 19 & 11 \\
\hline 13 & 24 & 24 & 34 & 17 \\
\hline 14 & 22 & 44 & 4 & 31 \\
\hline 15 & 55 & 6 & 4 & 35 \\
\hline 16 & 36 & 21 & 24 & 19 \\
\hline 17 & 34 & 15 & 33 & 17 \\
\hline 18 & 12 & 9 & 72 & 7 \\
\hline 19 & 45 & 3 & 41 & 12 \\
\hline 20 & 30 & 27 & 22 & 22 \\
\hline
\end{tabular}

Con respecto a las propiedades organolépticas de los ácidos y las bases (ítems 2, 6, 8), en el ítem 2 se evidencia que un 93 $\%$ de los estudiantes asocia los sabores amargo y agrio al concepto de ácido, el 5\%, considera que el sabor salado hace parte de los ácidos y un porcentaje muy bajo (2\%) lo relaciona al sabor dulce. Se concluye que la mayoría de los estudiantes correlaciona adecuadamente, ya sea por información previa o por construcción intuitiva, la propiedad organoléptica del sabor con el concepto de ácido.

Del ítem 6, un $45 \%$ de los encuestados asocia la sensación resbalosa con las propiedades de una sustancia básica, pero la sensación áspera también es vinculada a este concepto por un $29 \%$ de los estudiantes. Las sensaciones abrasiva y rugosa también fueron asociadas con la presencia de una sustancia básica ( 11 y $15 \%$ respectivamente). En el ítem 8 , se observa que el $13 \%$ de los estudiantes asocia las bases con el sabor amargo, mientras que otros consideran los sabores dulce y salado para estas sustancias, pues un $78 \%$ de los estudiantes señaló las respuestas a estos sabores (39\% en cada caso). 9\% considera que el sabor agrio está presente en las sustancias básicas. Puede decirse que en la población estudiada existe una fuerte creencia de que las bases tienen un sabor dulce o salado.

Los ítems 4 y 10, recogen información sobre la composición química y la nomenclatura de los ácidos y de las bases. $43 \%$ de los estudiantes claramente no establece relación entre el concepto de ácido su la fórmula química, confundiendo su fórmula con la de una base. Sólo el $23 \%$ de los estudiantes correlacionó la composición química con el concepto de ácido. En cuanto a las bases, el $37 \%$ de los estudiantes asocia este término con la formula correspondiente. Esto revela la carencia de conceptos fielmente estructurados, sobre ácidos y bases, desde una perspectiva química, evidentemente por la formación inicial y primaria de los estudiantes, pero también indica que es necesario emprender la construcción adecuada y completa de estas temáticas involucrando los niveles simbólicos (nomenclatura química) y submicroscópicos (composición atómica).

Con respecto a las reacciones que presentan los ácidos y las bases (ítems 1, 3, 9, 17, 20), la mayoría de los estudiantes NO posee información clara sobre la formación de ácidos y bases: los ítems 17 y 20 muestran que el $17 \%$ de los estudiantes asocia la formación de un óxido básico con los elementos metálicos, lo cual es correcto, pero el resto $(83 \%)$ lo asocia con elementos no metálicos, lo cual es incorrecto. Sólo un $22 \%$ de los estudiantes asoció la formación del óxido ácido con los elementos no metálicos, de allí que el 78\% lo asocie a elementos metálicos. Lo anterior pone en evidencia la necesidad de incorporar conceptualmente las reacciones generales de formación de ácidos y bases desde un esquema periódico, incluyendo la formación de los óxidos y sus reacciones con agua. 
Los ítems 1, 3, 9, que examinan la reactividad de los ácidos y las bases, muestran puntuaciones por debajo del $40 \%$ hacia las respuestas correctas, lo que hace evidente que la mayoría de los estudiantes NO identifica los productos generales de descomposición de ácidos y de bases. Según esta información, es necesario incorporar la descripción de reacciones ácido-base y redox sencillas en la construcción de la temática.

En los ítem 7, 12, 18 y 19, se observa que el $72 \%$ de los estudiantes identifica las propiedades ácidas o básicas presentes en algunos materiales y alimentos de uso cotidiano. Esto es importante, porque presupone ciertas aplicaciones prácticas, generando aprendizaje significativo sobre este tema.

En las respuestas 11, 13, 14 y 16 que corresponden al reconocimiento de la importancia de los ácidos y de las bases en el cuerpo humano, es evidente la dificultad en reconocer la presencia de sustancias ácidos en los procesos biológicos. En la pregunta 11, el $68 \%$ de los estudiantes asoció la acidez estomacal a compuestos químicos diferentes a la presencia de ácido clorhídrico. En la respuesta 13, se ratifica dicha dificultad. Además, existe un buen porcentaje de estudiante que reconoce la presencia de urea, en la orina, como compuesto básico (44\%, ítem 14).

El ítem 16 revela la problemática de no identificar el tipo de ácido que está presente en los diferentes procesos bioquímicos a nivel muscular. Sólo el $24 \%$ relaciona estos procesos con el desprendimiento de ácido láctico en los músculos. Los resultados de los ítems 11, y 13 a 16, indican claramente que es necesario integrar los conceptos de acidez y basicidad con procesos biológicos fundamentales. Esto además permitirá superar la perspectiva química y generar significado biológico integral.

Del ítem 15, el $55 \%$ de los estudiantes reconoce una sustancia básica (bicarbonato de sodio) en la fabricación de alimentos. Este resultado es importante porque muestra que la mayoría de los estudiantes logra vincular adecuadamente una sustancia de carácter básico en procedimientos industriales, permitiendo nuevamente la integración del concepto de bases con el mundo práctico.

\section{CONCLUSIONES}

Se realizó una recopilación histórico-epistemológica de los conceptos de ácido y base que se han manejado hasta nuestros días, de forma resumida y concreta, que puede ser empleada como marco conceptual inicial para la enseñanza de este tema. Para los estudiantes fue más fácil identificar las sustancias básicas que las acidas, por su nomenclatura y por su composición química. Se observó la necesidad de integrar los conceptos de acidez y basicidad con procesos biológicos fundamentales. El cuestionario aplicado permitió obtener información sobre las ideas de la población estudiada, con respecto a los ácidos y las bases, lo cual es de gran utilidad para el diseño curricular y la elaboración de estrategias didácticas que permitan mejorar la enseñanza de la química. Los datos podrán compararse más adelante con los resultados de otros entornos sociales.

\section{AGRADECIMIENTOS}

Los autores agradecen a la Facultad de Ciencias de la Universidad Nacional de Colombia y a la institución Colegio Brazuelos de la localidad quinta de USME (BogotáColombia), por su apoyo logístico.

\section{REFERENCIAS}

[1]. MINISTERIO DE EDUCACIÓN NACIONAL (MEN) (2004), Formar en ciencias: el desafío. Estándares básicos de competencias en ciencias naturales y ciencias sociales. Serie Guías No. 7. Bogotá.

[2]. C. Furió, M. Calatayud, S. Bárcenas, "Surveying students' conceptual and procedural knowledge of acidbase behavior of substances", Journal of Chemical Education, vol. 84(10), p.p. 1717-1724, 2007.

[3]. H. Leicester, Panorama histórico de la química, Editorial Alambra: Madrid, 1967.

[4]. I. Asimov, Breve historia de la química, Alianza Editorial: Estados Unidos, 2003.

[5]. J. Partington, Historia de la química, Espasa - Calpe: Buenos Aires (Argentina), 1945.

[6]. R. Jiménez, E. de Manuel, "La Neutralización ácido-base a debate", Enseñanza de las Ciencias, vol. 20 (3), p.p. 451-464, 2002.

[7]. G. E. Rodgers, Descriptive Inorganic, Coordination, and Solid State Chemistry, Second Edition, Thomson Learning: Toronto, 2002.

[8]. G. Wulfsberg, Inorganic Chemistry, University Science Books: U.S.A, 2000.

[9]. P. Atkins, T. Overton, J. Rourke, M. Weller, F. Armstrong, Química Inorgánica, Edición 4, McGrawHill: China, 2008.

[10]. J. Campanario, J. Otero, "Más allá de las ideas previas como dificultades del aprendizaje: las pautas de pensamiento, las concepciones epistemológicas y las estrategias metacognitivas de los alumnos de ciencias", Enseñanza de las ciencias, vol. 18(2), p.p. 155-169, 2000.

[11]. H. Ozmen, "Some Student Misconceptions in Chemistry: A Literature Review of Chemical Bonding", Journal of Science Education and Technology, vol. 13, p.p. 147-159, 2004.

[12]. J. Lin, M. H. Chiu, "Exploring the Characteristics and Diverse Sources of students' mental models of acids and bases". International Journal of Science Education, vol. 29(6), p.p. 771-803, 2007.

[13]. C. Furio, C. Furio, "Dificultades conceptuales y epistemológicas en el aprendizaje de los procesos 
químicos”, Educación química, vol. 11(3), p.p. 300-308, 2000.

[14]. M. Jiménez, E. Torres, F. Salinas, "Las concepciones alternativas de los estudiantes universitarios sobre los procesos ácido-base", Journal of Science Education, vol. 3(1), p.p. 18-24, 2002.

[15]. B. Yezdan, "Turkish prospective chemistry teachers' alternative conceptions about acids and bases", School Science and Mathematics, vol. 109(4), p.p. 212-222, 2009.

[16]. R. Artdej, T. Ratanaroutai, R. Coll, T. Thongpanchang, "Thai Grade 11 students' alternative conceptions for acid-base chemistry", Research in Science and Technological Education, vol. 28(2), p.p. 167-183, 2010.

[17]. L. McClary, V. Talanquer, "College chemistry students' mental models of acids and acid strength", Journal of Research in Science Teaching, vol. 48, p.p. 396-413, 2011.

[18]. M. Çalik, A. Ayas,, R. Coll, "Investigating the Effectiveness of Usage of Different Methods Embedded with Four-Step Constructivist Teaching Strategy", Journal of Science Education and Technology, vol. 19(1), p.p. 32-48, 2010.

[19]. Cokelez, A., "A comparative study of French and Turkish students' ideas on acid-base reactions". Journal of Chemical Education, vol. 87(1), p.p. 102-106, 2010.

[20]. S. Matute, "Concepciones de los estudiantes sobre las sustancias ácidas y básicas”, Educación y Humanismo, vol. 13, p.p. 17-33, 2011. 